\title{
Hipertrofia septal assimétrica: técnica de correção e análise da evolução pós-operatória
}

Fábio B. JATENE*, Marcelo B. JATENE*, José Otávio C. AULER JÚNIOR*, Marluce de Oliveira e SILVA*, Fúlvio PILEGGI*, Miguel BARBERO-MARCIAL*, Geraldo VERGINELLI*, Adib D. JATENE*

RBCCV 44205-144

JATENE, F. B.; JATENE, M. B.; AULER JÚNIOR, J. O. C.; SILVA, M. O.; PILEGGI, F.;BARBEROMARCIAL, M.; VERGINELLI, G.; JATENE, A. D. - Hipertrofia septal assimétrica: técnica de correção e análise da evoluçäo pós-operatória. Rev. Bras Cir. Cardiovasc., 6 (3) 147-152, 1991.

RESUMO: A hipertrofia septal assimétrica (HSA), ou estenose subaórtica dinâmica é doença que apresenta características peculiares relacionadas tanto ao diagnóstico quanto à técnica cirúrgica empregada para o seu tratamento. De setembro de 83 a setembro de 89,16 pacientes consecutivos ( 10 homens) com idades de sete meses a 66 anos $(30,87 \pm 22,42)$ foram submetidos a tratamento cirúrgico. Este consistiu, fundamentalmente, da não abordagem do septo interventricular e sim da ressecção muscular realizada na região da via de saída do ventrículo esquerdo, parede anterior entre os seios aórticos coronarianos direito e esquerdo. Esta miectomia, profunda e extensa foi realizada isoladamente em dez pacientes e nos outros seis associada a outros procedimentos: ligadura de canal arterial e ressecção de anel subaórtico fibroso em dois; descalcificaçäo de valva aórtica (VAo) em um; ressecção de banda muscular em ventrículo direito em um: ressecçäo de vegetaçäo de VAo em um; fechamento de CIV com plastia de VAo em um. Em todos os casos foram feitas medidas intra-operatórias das pressōes do VE e aorta após desconexão da circulação extracorpórea. Nảo houve nenhum óbito imediato neste grupo de pacientes. Houve um $(6,2 \%)$ óbito tardio, um anos após, não relacionado à doença. Com relação a distúrbios na condução atrioventricular, houve um $(6,2 \%)$ bloqueio $A-V$ total, com implante de marcapasso definitivo. Houve dois $(12,5 \%)$ bloqueios de ramo esquerdo, e três $(18,7 \%)$ bloqueios divisionais ânterosuperiores, sem alteraçōes nos demais pacientes. Em um peíodo de 19 a 90 meses $(56,00 \pm 23,72), 14$ $(87,5 \%)$ pacientes encontram-se assintomáticos.

DESCRITORES: estenose subaórtica hipertrófica, cirurgia.

\section{INTRODUÇĀO}

A hipertrofia septal assimétrica (HSA) caracteriza-se por uma estenose subaórtica de características peliculares. Fundamentalmente, essa obstrução da via de saída do ventrículo esquerdo se deve a uma hipertrofia anômala do septo interventricular e que durante a sístole se aproxima da mitral, obstruindo a via de saída do ventrículo esquerdo (VE). Desta forma, essa obstrução é dinâmica e portanto diferente daquelas que ocorrem por presença de fibrose na porção sub aórtica e que são consideradas estenoses fixas.

Essa doença recebeu uma denominação muito vasta e se considera que mais de 60 nomes foram usados nos vários centros para denominar a mesma lesão ${ }^{6}$. Entre os seus nomes mais conhecidos destacam-se, além de HSA: estenose subaórtica dinâmica ou hipertrófica ou muscular e estenose sub aórtica hipertrófica idiopática, entre muitos outros.

A sintomatologia referida geralmente é a mesma

Trabalho realizado no Instituto do Coração do Hospital das Clínicas da Faculdade de Medicina da Universidade de São Paulo. São Paulo, SP, Brasil. Apresentado ao $18^{\circ}$ Congresso Nacional de Cirurgia Cardíaca. Rio de Janeiro, RJ, 5 e 6 de abril, 1991.

* Do Instituto do Coração do Hospital das Clínicas da Faculdade de Medicina da Universidade de São Paulo.

Endereço para separatas: Fábio Jatene. Av. Dr. Enéas Carvalho de Aguiar, 44. Divisão Cirúrgica. 05403 São Paulo, SP, Brasil. 
JATENE, F. B.; JATENE, M. B.; AULER JÚNIOR, J. O. C.; SILVA, M. O.; PILEGGI, F.;BARBERO-MARCIAL, M.; VERGINELLI, G.; JATENE, A. D. - Hipertrofia septal assimétrica: técnica de correção e análise da evolução pós-operatória. Rev. Bras Cir. Cardiovasc., $6(3): 147-152,1991$.

que dos casos de estenose aórtica, muito embora, possam estar presentes sintomas associados à presença de insuficiência mitral, pela compressão e disfunção do aparelho subvalvar mitral pela porção hipertrofiada do septo interventricular.

A indicação cirúrgica se faz em cerca de um terço dos pacientes, sobretudo naqueles com maior repercussão hemodinâmica ${ }^{3,4}$.

Este trabalho visa à avaliação de aspectos técnicos e evolutivos da cirurgia em um grupo de pacientes operados no Instituto do Coração, para correção dessa enfermidade.

\section{CASUÍSTICA E MÉTODOS}

De setembro de 83 a setembro de 89,16 pacientes consecutivos, sendo $10(62,5 \%)$ masculinos, com idade entre sete meses e 66 anos $(30,87 \pm$ $22,42)$ foram submetidos a tratamento cirúrgico de HSA

Após suspeita clínica, o diagnóstico foi confirmado em todos os pacientes pela realização de ecocardiograma bidimensional e estudo hemodinâmico.

O gradiente VE/Ao, medido em todos os pacientes por cateterismo, exceto em dois deles onde a medida foi realizada por eco-Doppler, revelou valores entre 30 e $125 \mathrm{mmHg}(82,4 \pm 34,16)$.

O tratamento cirúrgico, que constitui na miectomia, foi realizado sob circulação extracorpórea, com o paciente a $28^{\circ} \mathrm{C}$ e cardioplegia cristalóide (SaintThomas). O volume de cardioplegia utilizado esteve entre 70 e $800 \mathrm{ml}(350,66 \pm 172,69)$ e os tempos de circulação extracorpórea entre 43 e $108 \mathrm{~min}$. $(63,87$ $\pm 21,12)$ e de pinçamento aórtico entre 32 e $64 \mathrm{~min}$. $(40,93 \pm 10,18)$. A abordagem foi realizada, por via transaórtica, sob forma de ressecção muscular, realizada na regiāo subaórtica sendo que não se buscava a correção direta da hipertrofia do septo interventricular. Essa ressecção, profunda e extensa, realizada preferencialmente com bisturi de lâmina 11, era feita junto à via de saída do VE na sua parede ânterolateral na região correspondente à projeção entre as válvulas aórticas coronarianas esquerda e direita e dirigida no interior do VE para a região anterior da base do músculo papilar anterior. Em alguns casos, essa ressecção era realizada com o auxílio de tesoura ou mesmo de pinça tipo "saca-bocados".

A miectomia foi realizada isoladamente em dez $(62,5 \%)$ pacientes e associada a outros procedimentos em seis $(37,5 \%)$. Estes procedimentos estão listados na Tabela 1 .

Após ressecção, a valva era avaliada para detecção

\section{TABELA 1}

\section{PROCEDIMENTOS ASSOCIADOS AO TRATA- MENTO CIRÚRGICO DA HSA.}

\begin{tabular}{|c|}
\hline 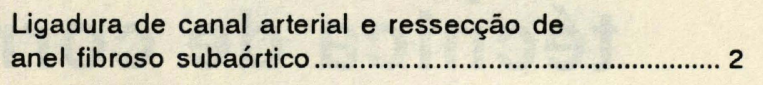 \\
\hline Descalcificação da valva aórtica \\
\hline Ressecção de banda muscular em VD ............................ 1 \\
\hline Retirada de vegetaçāo em aorta ...................................... 1 \\
\hline Fechamento de CIV + plastia de valva aórtica ............. 1 \\
\hline
\end{tabular}

de possíveis lesões nas válvulas e, a seguir, a aorta era fechada, o ar retirado e os batimentos restabelecidos. Após estabilização e já com o paciente fora de circulação extracorpórea, eram realizadas medidas pressóricas, com o auxílio de transdutor, para avaliação da pressão do VE e da aorta ascendente, na busca de possíveis gradientes pressóricos. Se não houvesse gradiente, ou se ele fosse pequeno e não significativo, as cânulas de perfusão eram retiradas.

\section{RESULTADOS}

Não houve nenhum óbito imediato neste grupo de pacientes.

Houve um $(6,2 \%)$ óbito tardio, cerca de um ano após a cirurgia, não relacionado com a doença.

Com relação aos distúrbios da conducão atrioventricular, os dados estão listados na Tabela 2.

\section{TABELA 2}

\section{DADOS REFERENTES AOS DISTÚRBIOS DA CONDUÇÃO ATRIOVENTRICULAR OBSERVA- DOS NO PÓS-OPERATÓRIO.}

\begin{tabular}{|c|c|}
\hline Bloqueio divisional ântero-superior .............. 3 & $(18,7 \%)$ \\
\hline Bloqueio de ramo esquerdo ......... & $(12,5 \%)$ \\
\hline Bloqueio AV total (com marc & 106 \\
\hline
\end{tabular}

Todos os demais pacientes não apresentaram quaisquer alterações de ritmo ou condução atrioventricular.

A evolução pós-operatória imediata revelou que um $(6,25 \%)$ paciente foi reoperado por sangramento para revisão de hemostasia. Ainda na UTI, três $(18,7 \%)$ pacientes evoluíran em fibrilação atrial aguda, com posterior reversão para ritmo sinusal. O implante definitivo de marcapasso foi realizado em um paciente no $23^{\circ}$ dia de pós-operatório. O tempo médio

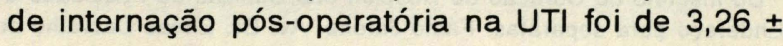


JATENE, F. B.; JATENE, M. B.; AULER JÚNIOR, J. O. C.; SILVA, M. O.; PILEGGI, F.;BARBERO-MARCIAL, M.; VERGINELLI, G.; JATENE, A. D. - Hipertrofia septal assimétrica: técnica de correçāo e análise da evoluçāo pós-operatória. Rev. Bras Cir. Cardiovasc., 6 (3):147-152, 1991.

3,06 dias e o tempo de internação até a alta hospitalar foi de $11,07 \pm 4,48$ dias.

A classificação funcional pré e pós-operatória dos pacientes pode ser avaliada na Tabela 3.

TABELA 3

CLASSE FUNCIONAL PRÉ E PÓS-OPERATÓRIA DOS PACIENTES

\begin{tabular}{lcc}
\hline & Pré-op. & Pós-op. \\
\hline CF I & - & 14 \\
CF II & 7 & 1 \\
CF III & 7 & - \\
CF IV & 2 & - \\
\hline
\end{tabular}

A evolução tardia, realizada entre 19 e 90 meses $(56,00 \pm 23,72)$, revelou que 14 pacientes se encontram no momento assintomático e um $(6,2 \%)$ paciente apresenta dispnéia aos médios esforços.

\section{COMENTÁRIOS}

O tratamento cirúrgico da HSA é, hoje, procedimento bem sistematizado e isto se deve em grande parte a MORROW et alii ${ }^{4}$ e Morrow ${ }^{6}$ que apresentaram várias contribuições a respeito de HSA. Esses autores relataram, recentemente, experiência a respeito de uma série e 342 pacientes com $9 \%$ de mortalidade imediata, devida a vários fatores, entre eles: imperfeiçōes técnicas na realização da ressecção muscular, falência ventricular esquerda, arritmias intratáveis e infarto do miocárdio. Na nossa experiência, embora com um número reduzido de casos, não se observou mortalidade hospitalar ou imediata e o único óbito ocorrido foi tardio e sem relação com a doença em questão. Com relação aos distúrbios de condução, consideraram que os bloqueios AV totais foram evitados na grande maioria dos casos pela utilização da técnica cirúrgica recomendada, que evita a abordagem do septo na região do nó $\mathrm{AV}$ e do sistema de condução.

Entretanto, na experiência dos autores citados, a ocorrência de bloqueio de ramo esquerdo é alta, ao redor de $85 \%$. Isto conduz a uma conclusão interessante, comentada por MORROW 6: "embora a ocorrência de bloqueio de ramo direito seja raríssima, quando ela está presente no pré-operatório, a possibilidade de bloqueio AV total aumenta muito, devido à grande incidência das lesöes sobre o ramo esquerdo do sistema de condução".

No nosso material, a ocorrência de bloqueios de ramo foi menor, sendo de $12,5 \%$ para o ramo esquerdo e de $18,7 \%$ para divisão ântero-superior. Em só um $(6,2 \%)$ caso ocorreu bloqueio AV total, necessitando implante de marcapasso definitivo, realizado no $23^{\circ}$ dia de pós-operatório.

Com relação ao aspecto técnico, um detalhe que deve ser enfatizado diz respeito ao exato local da ressecção da hipertrofia septal. Como preconizado pelos mesmos autores e COOPER et alii ${ }^{1}$, o local da ressecção deveria se situar imediatamente abaixo da correspondente projeção do $1 / 3$ médio da válvula aórtica coronariana direita. Entretanto, a abordagem neste ponto, embora levando ao alívio da estenose pode determinar maior número de complicaçōes, relacionadas não só aos distúrbios de condução como também à ocorrência de comunicaçöes interventriculares (CIV) agudas ou tardias. $\mathrm{Na}$ nossa experiência, o local de alívio da estenose deveria se situar em um ponto próximo do preconizado por MORROW ${ }^{6}$, porém um pouco mais em direção à parede livre do $V E$, ou seja, abaixo da comissura aórtica coronariana esquerda/direita. Desta forma procura-se evitar a abordagem direta do septo reduzindo-se ao máximo a ocorrência de complicacōes daí resultantes, como distúrbios de condução ou CIV. Da mesma maneira a tática por nós utilizada promove uma ampla abertura na região subaórtica e um perfeito alívio da estenose ai presente, por "quebrar" ou interromper a continuidade da hipertrofia septal com a parede ventricular livre do VE.

A respeito da evolução tardia, MARON et alii ${ }^{3}$ mostraram que embora a maioria dos pacientes operados na sua série tenha apresentado evolução favorável, cerca de $12 \%$ deles apresentaram deterioração clínica em evolução de cinco anos, além de pequena mas contínua mortalidade anual. No nosso material, embora com menor cauística, numa evolução média de 56 meses não houve piora clínica e nem mortalidade tardia que pudesse ser atribuída à evolução da doença.

Com relação à presença de insuficiência mitral associada à HSA, esta é uma ocorrência relativamente freqüente e que pode ser atribuída à compressão e conseqüente disfunção do aparelho subvalvar mitral pela hipertrofia septal. STANLEY \& RANKIN ${ }^{10}$ relatam a utilidade do ecocardiograma trasnsesofágico e intra-operatório na avaliação de pacientes portadores de insuficiência mitral e HSA. No nosso material a insuficiência mitral esteve presente em oito pacientes, sendo discreta em um, moderada em seis e severa em um. Em nenhum caso a valva mitral foi substituída ou submetida a plastia. Em seis casos houve resolução do problema com a miectomia e em dois casos a insuficiência mitral remanescente era de gráu discreto, sem repercussão clínica. Outro dado interessante, relatado 
JATENE, F. B.; JATENE, M. B.; AULER JÚNIOR, J. O. C.; SILVA, M. O.; PILEGGI, F.;BARBERO-MARCIAL, M.; VERGINELLI, G.; JATENE, A. D. - Hipertrofia septal assimétrica: técnica de correção e análise da evolução pós-operatória. Rev. Bras Cir. Cardiovasc., 6 (3):147-152, 1991.

por SHAH et alii ${ }^{8,9}$ através de estudo ecocardiográfico, diz respeito à insuficiência mitral na HSA que seria detectada pela observação do movimento sistólico da cúspide anterior da mitral. Eles concluíram que seriam necessárias alteraçōes de coaptação do cúspide anterior da mitral para promover o aparecimento de tal movimento.

Embora os princípios básicos da técnica cirúrgica já estejam estabelecidos há anos, ROBICSEK \& DAUGHERTY ${ }^{7}$ propuzeram, recentemente, um dispositivo para facilitar a correção cirúrgica.

Tal instrumento, semelhante a uma cureta, apresenta um pequeno recipiente na sua extremidade, acoplado à lâmina cortante, que se encaixa na regiāo hipertrofiada e por tração promove a ressecção da zona hipertrofiada. O dispositivo usado em alguns pacientes propriciou a ressecção da hipertrofia muscular com bons resultados e de uma maneira segura e confiável. Nos nossos casos foram utilizados preferencialmente o bisturi com lâmina 11, além de pinça "saca-bocados", quando necessário, também com bons resultados.

STULZ et alii ${ }^{11}$ relatam um caso de HSA e vários episódios recorrentes de endocardite e que foi submetida a tratamento cirúrgico. Na nossa casuística um paciente apresentava lesōes vegetantes, e que foram ressecadas, nas válvulas da valva aórtica, devidas também a um episódio prévio de endocardite.

Embora na nossa casuística, a média de idade estivesse ao redor dos 30 anos, COOPER et alii ${ }^{1}$ e $\mathrm{KOCH}$ et alii $^{2}$ fizeram referência a pacientes de uma faixa etária elevada e que foram operados com bons resultados além de melhora sintomática e hemodinâmica acentuadas, concluindo não ser a idade avançada uma contra-indicação para este procedimento.

Por fim, concluímos que o tratamento cirúrgico da HSA na forma como foi realizado, produziu bons resultados, que se mantiveram a longo prazo, além de pequeno número de complicaçōes severas.

RBCCV 44205-144

JATENE, F.B.; JATENE, M.B.; AULER JÚNIOR, J.O.C.; SILVA, M.O; PILEGGI, F.; BARBERO - MARCIAL, M.; VERGINELLI, G.; JATENE, A.D. - Dynamic subaortic stenosis: surgical treatment and postoperative evaluation. Rev. Bras. Cir. Cardiovasc., 6(3):147-152, 1991.

ABSTRACT: The dynamic subaortic stenosis, more commonly named hypertrofic subaortic stenosis (HSS) presents some aspects specially related with its diagnosis and surgical treatment. From September/ 83 to September $/ 89$, sixteen patients $(10 \mathrm{men}$ ) with age between 7 month and 66 years $(30.87 \pm 2.42)$ were submitted to surgical treatment to correct the HSS. The surgical technique employed the muscular resection in left ventricular outflow tract, in the anterior wall, between left and right coronary aortic sinus. This resection was characterized by an extensive and deeply miectomy and was performed with no other procedures in 10 patients and in association with other procedures in 6 patients (correction of patent arterious ductus with fibrotic subaortic stenosis in 2; surgical conservative procedure in aortic valve stenosis in one; right ventricle muscular anomalous band resection of vegetative lesion in aortic leaflet in one; interventricular septum communication closure and aortic valve conservative procedure in one. After cardiopulmonary by-pass, intraoperative evaluation in aortic and left ventricle pressure were made in all patients. No immediate deaths were present in this group of patients. There was one $(6.2 \%)$ death with one year of follow-up, not related with the disease. After surgery related to AV disturbances, one $(6.2 \%)$ patient presented AV block with need of pacemaker implantation; two (12.5\%) presented left bundle block three $(18.7 \%)$ presented left bundle branch block, with no alteration in the other patients. In the late followup, between 19 and 90 months $(56.00 \pm 23.72), 14(87.5 \%)$ patients are with no symptoms.

DESCRIPTORS: hypertrofic subaortic stenosis, surgery.

\section{REFERÊNCIAS BIBLIOGRÁFICAS}

1 COOPER, M.M.; McINTOSH, C. L.; TUCKER, E., CLARK, R. E. - Operation for hypertrophic subaortic stenosis in the aged. Ann. Thorac. Surg., 44: 370-378, 1987. A. G. - Results of operation for obstructive hypertrophic cardiomyopathy in the elderly. Am. J. Cardiol., 46: 1963-1966, 1980.
MARON, B. J.; MERRILL, W. H.; FREIER, P. A.; KENT, K. M.; EPSTEIN, S. E.; MORROW, A. G. - Long-term clinical course and symptomatic status of patients after operation for hypertrophic subaortic stenosis. Circulation, 57: 1205-1213, 1978.

4 MORROW, A. G.; REITZ, B. Z.; EPSTEIN, S. E. - Operative treatment in hypertrophic subaortic stenosis: techniques, and the results of pre and postoperative assessment in 83 patients. Circulation, 52: 88-102, 1975. 
JATENE, F. B.; JATENE, M. B.; AULER JÚNIOR, J. O. C.; SILVA, M. O.; PILEGGI, F.;BARBERO-MARCIAL, M.; VERGINELLI, G.; JATENE, A. D. - Hipertrofia septal assimétrica: técnica de correção e análise da evoluçäo pós-operatória. Rev. Bras Cir. Cardiovasc., 6 (3):147-152, 1991.

5 MORROW, A. G. - Hypertrophic subaortic stenosis: operative methods utilized to relieve left ventricular obstrucion. J. Thorac. Cardiovasc. Surg., 76: 423430, 1978.

6 MORROW, A. G. - Hypertrophic subaortic stenosis. In: JAMIESON, S. W. \& SHUMWAY, N. E. (eds.). Operative surgery 4. ed. Saint Louis, The C V Mosby Company, 1986. p. 335-345.

7 ROBICSEK, F. \& DAUGHERTY, H. K. - A new instrument to facilitate myectomy in subaortic hypertrophic stenosis. J. Thorac. Cardiovasc. Surg., 95: 533-534, 1988.

8 SHAH, P. M.; GRAMIAK, R.; ADELMAN, A. G.; WIGLE, E. D. - Role of echocardiography in diagnostic and hemodynamic assesment of hypertrophic subaortic stenosis. Circulation, 44: 891-898, 1971.

9 SHAH, P. M.; TAYLOR, R. D.; WONG, M. - Abnormal mitral valve coaptation in hypertrophic obstructive anterior motion of mitral valve. Am. J. Cardiol., 48: 258-262, 1981.

10 STANLEY, T. E. \& RANKIN, J. S. - Idiopathic hypertrophic subaortic and Ischemic mitral regurgitation: the value of intraoperative transesophageal echocardiography and Doppler color flow imaging in guiding operative therapy. Anesthesiology, 72: 10831085, 1990.

11 STULZ, P.; ZIMMERLI, W.; MIHATSCH, J.; GRADEL, E. - Recurrent infective endocarditis in idiopathic hypertrophic subaortic stenosis. Thorac. Cardiovasc. Surg., 37: 99-102, 1989.

\section{Discussão}

\section{DR. RÉGIS JUCÁ}

Fortaleza, CE

Congratulo-me com o Dr. Fábio Jatene e colegas do InCor, pela oportuna escolha deste controvertido tema, que sempre desperta interesse, pelos seus múltiplos aspectos, entre os quais: quando operar e como operar. O nome hipertrofia septal assimétrica é o mais usado por certos grupos, mas estenose hipertrófica idiopática subaórtica e miocardiopatia hipertrófica obstrutiva são também aceitos. Aliás, mais de 50 denominaçōes ou títulos já foram catalogados para esta doença. Jatene e associados, do InCor, adotaram a técnica mais empregada, que é a miomectomia, estudada e popularizada por Morrow, do National Institute of Health, de Bethesda, USA. Este grupo operou 342 pacientes em um período em que 1312 casos foram diagnosticados com indicação cirúrgica de $26 \%$, portanto. Dos três achados mais característicos da doença, ou sejam: hipertrofia septal assimétrica, desorganiza- ção das miofibrilas (ambas apresentam variados padrōes) e o movimento anterior sistólico da mitral este último tem grande implicação terapêutica, porque a valva mitral, encostando no septo interventricular, contribui consideravelmente para a obstrução. Tal achado foi enfatizado, inicialmente, por Robert Leachman, de Houston, no início dos anos 70, logo após o emprego do ecocardiograma (O Eco tem um enorme papel no manejo desta doença). Como conseqüência, Cooley realizou a substituição da valva mitral (SVM) por uma prótese de baixo perfil, em alguns pacientes, com completo desaparecimento dos gradientes sistólicos. Morrow reagiu enfática e fortemente a essa alternativa. Entretanto, Mclntosh, sucessor e discípulo de Morrow, no N.I.H., após sua morte realizou substituiçäo da valva mitral em aproximadamente $25 \%$ de 261 doentes operados. A percentagem é quase igual à de Cooley (29\%). Em outra publicação de Mclntosh, em 156 casos realizou 108 miomectomias e 48 SVM Mclntosh apoiado, em dados de ecocardiografia, cita quatro critérios para a substituição da mitral como forma de tratamento: 1) septo interventricular com espessura menor do que $18 \mathrm{~mm}$; 2) morfologia septal atípica; 3) miomectomia prévia; 4) doença mitral orgânica. O Eco desempenha um papel importante no pré e no transoperatório para decidir o melhor tipo de conduta na experiência do N. I. H. Walker e colaboradores, de Edinburgh, Escócia, operaram 22 pacientes divididos em dois grupos de 11 - um tratado com miomectomia e outro com SVM e concluem que a SVM oferece um resultado mais previsível e é o procedimento de escolha nos centros com menor experiência (Ann. Thorac. Surg., 48: 528-535, 1989. Em Editorial no mesmo número da revista, Mclntoch concorda em parte com as conclusōes de Walker et alii. O Dr. Fábio Jatene relatou que a insuficiência mitral desapareceu após a miomectomia em todos os casos o que também aconteceu na experiência da Mayo Clinic. Nossa experiência é muito limitada com os dois tipos de cirurgia, mas concordo com o que Kirklin escreveu no seu livro sobre algumas dificuldades na SVM. Penso que elas podem ser minimizadas usando a via transeptal. Feitas estas considerações, ao mesmo tempo em que parabenizo os autores por trazerem a nós a experiência do InCor, indago: 1) Qual o papel do Eco no pré e no transoperatório na rotina do InCor. 2) Qual a opinião sobre a SVM nesta doença. 3) A cirurgia evita ou diminui a incidência de morte súbita? Agradeço a oportunidade de comentar este excelente trabalho.

\section{DR. NILZO RIBEIRO \\ Salvador, $B A$}

Assim como várias têm sido as designações para esta doença, também um grande número de propostas cirúrgicas tem sido tentado, demonstran- 
JATENE, F. B.; JATENE, M. B.; AULER JÚNIOR, J. O. C.; SILVA, M. O.; PILEGGI, F.;BARBERO-MARCIAL, M.; VERGINELLI, G.; JATENE, A. D. - Hipertrofia septal assimétrica: técnica de correçāo e análise da evolução pós-operatória. Rev. Bras Cir. Cardiovasc., 6 (3):147-152, 1991.

do uma insatisfação quanto aos resultados. A opção proposta por Morrow tem se mostrado a melhor. O trabalho apresentado pelo Dr. Fábio Jatene mostra excelentes resultados. As alteraçōes do ritmo cardíaco com baixa incidência de bloqueios devem estar relacionadas à modificação do local da infundibulectomia como mostrado pelos autores. Gostaríamos de saber se na criança de sete meses operada havia, ou não, defeitos associados, pois, quando essa associação ocorre, os gradientes transventriculares tendem a diminuir ou, até mesmo, desaparecer. Existe, ainda, maior incidência em filhos de mães diabéticas. Quanto ao gradiente indicativo da cirurgia, notamos pacientes com $30 \mathrm{~mm}$. Esses pacientes foram estimulados? Como se comportou o gradiente? Quanto ao intra-operatório, quais os valores de gradiente pequenos e/ou não significativos que poderiam ser tolerados? Em relação à evolução a longo prazo, essa é uma preocupação de todos, pois a doença é uma forma de miocardiopatia. Em nossa experiência, dois pacientes foram reestudados com dois e três anos de pós-operatório. O primeiro, mesmo com infusāo de inotrópico, não mostrava gradiente sistólico e o segundo, um gradiente de $7 \mathrm{mmHg}$. 0 seguimento a longo prazo desse grupo de pacientes traria uma grande contribuição que, tenho certeza, nos será dada pelo Dr. Fábio.

\section{DR. FÁBIO JATENE (Encerrando)}

Mais uma vez agradeço à Comissão Organizadora a oportunidade da apresentaçāo deste trabalho, bem como aos comentários dos Drs. Régis Jucá e Nilzo Ribeiro. Inicialmente, com relação às observaçōes do Dr. Régis, as considero muito pertinentes e agradeço o retrospecto que fez, o que complementa o nosso trabalho e valoriza o assunto em questão. Quanto às suas questōes, o ecocardiograma de rotina, na nossa instituição, tem um papel muito ativo, tanto no pré, quanto no transoperatório e mesmo no pósoperatório imediato e tardio, não só nesta doença, tanto para confirmação diagnóstica, quanto para avaliação da correção e da evolução. Com relação à substituição da valva mitral, embora reconhecendo os trabalhos citados, enfatizo que, no nosso material, a ressecção muscular certamente reduziu ou eliminou a compressão sobre o aparelho subvalvar mitral, o que proporcionou o retorno desta valva à sua condição normal e resolução da insuficiência. Em nenhum dos nossos pacientes foi necessária a substituição da mitral e em todos houve redução do processo, de tal forma que os sintomas foram eliminados. O tratamento da disfunção aórtica e, às vezes, da mitral, sem dúvida reduz a ocorrência das complicaçōes daí decorrentes. Entretanto, foi por nós citada a possibilidade de cirurgia promover bloqueios atrioventriculares, em maior ou menor grau, na dependência da técnica cirúrgica empregada com as conseqüências daí decorrentes. Respondendo aos comentários do Dr. Nilzo, a paciente com 7 meses apresentava outros defeitos associados e que foram listados em uma tabela apresentada. Com relação aos gradientes VE/Ao, embora tivéssemos valor mínimo de $30 \mathrm{mmHg}$, isto năo foi a regra, pois a média dos valores foi de $82 \mathrm{mmHg}$, valor bem acima do mínimo. Além disto, neste caso, o gradiente não teve participação decisiva na indicação, pois os sintomas eram mais relacionados à presença da insuficiência mitral. Quanto ao gradiente resifual, quando presente, ele era baixo, menor que $20 \mathrm{mmHg}$. Mais uma vez, gostaria de agradecer a oportunidade. Obrigado. 